\title{
The past, present, and future role of pediatricians
}

Medicine may be defined as a science that studies the diseases affecting humans, their prevention, and treatment to seek a cure. This definition may be construed as considering medicine part of biological sciences. Medicine as a science has benefited from a huge volume of knowledge in the past 40 years. We currently have the technical resources to diagnose an impressive number of diseases, but we are yet not capable of curing most of them.

The structure of "medical thinking" has changed with the introduction of new biological knowledge enabled through the understanding of pathophysiological mechanisms and the resulting possibility or opportunity of proposing or researching novel therapeutic approaches. Such ability to adapt our mental mechanisms to the huge volume of new knowledge is increasingly closer to be replaced by "artificial intelligence". We may easily imagine a computer where we type in a list of symptoms and physical, laboratory or radiological signs and it will provide a list of potential diagnoses and may even make a definitive diagnosis.

Considering technological advances, the immediate and medium-term future forces us to reflect on our professional practice. Such exercise allows us to become aware that medical practice is not limited to the mere implementation of biological knowledge, i.e. the development of our "scientific brain", which is extremely rich in frequently abstract data and swollen in the beginning of our profession. Experience and a close contact with our patients and, in the field of pediatrics, with the families that have trusted us with their children's health, contribute to the development of a "humanistic brain". These two brains, as defined by philosopher Michel Serres (Grand Rounds of CHU Sainte-Justine, September $\left.20^{\text {th }}, 2003\right)$, are complementary and require ongoing fostering and a persistent critical analysis.

The development of a humanistic brain takes place in the clinical setting, in the relationship we establish with our patients and their families. Creating a bond with a smooth, honest communication that will allow the development of mutual trust requires the health care provider to analyze their own values, prejudices, and beliefs, which should never have an impact on or become patent in the relationship with their patients. We should also prevent our mood and our experiences before the visit from influencing it. Let us not forget that listening to parents, establishing a dialog with our patients, and making an effort to understand their personal history will provide us with the indispensable elements to build a "therapeutic alliance". They also get to the clinical setting with their own values, beliefs, and prejudices, which should be respected and should not interfere with a relationship that aims to be therapeutic, based on the satisfaction of those involved in the dialog and avoiding unnecessary conflicts.

The first step in these visits is answering the questions that triggered the consultation, which will not always be in line with the physician's observations. For example, the parents of an infant are worried about their baby's frequent regurgitation, but the pediatrician notices an abdominal mass; listening to parents and providing an answer to their queries will allow to subsequently propose studying the tumor. We should never forget that the main goal is building a "therapeutic alliance" with the patients and their families; compliance with research proposals and eventual treatment depend on the trust developed among those involved.

The human relationship has always been the key to a successful clinical medicine; it accounts for the sphere of medicine that can never be replaced by technology. Although in recent years many of our colleagues have reduced the time they spend speaking with their patients, either due to financial reasons or the increased number of visits, we should go back to appreciating "empathy" as the critical element in our healing or restorative activity. Taking charge, accepting, sharing, accompanying are frequent expressions in the clinical setting; we should not forget that, in all cases, they embody an attitude towards a worried or suffering family, who seeks our advice with hope, looking for understanding, relief, and, potentially, solutions.

The physician-patient and family relationship currently takes place in a bioethical context that has developed in line with technological advances. Children and their families are considered members of the team, they share exchanges and take part in decision-making; of course, such dialog should be appropriate for the patient's age, level of understanding, 
and development. These principles respect the Convention on the Rights of the Child, which Argentina endorsed in October 1990 through Law no. 23849. Children and adolescents will take us by surprise with their questions, their ability to adapt to disease, their collaboration, and the great resilience they display in the relationship with their pediatricians.

We are now living a particularly difficult period in terms of physical, psychological, and social health. The pandemic has transformed our lives and mainly affected the way we interact with one another. The distance, hiding our faces behind a mask, and words which now travel through technological means, including screens, have deeply transformed our communication and the possibilities of getting closer. Telemedicine, whose use has increased in the current situation, although practical, will never replace a faceto-face meeting. It is worth noting that, in the "clinical space", communication can be verbal and non-verbal, but, in addition, the place where this visit occurs has a greater influence on message transmission or reception. It is not the same to talk in a hall or an office or behind open or closed doors, with a desk between us or around a round table, and the location also matters, whether it is a hospital, a clinic, a private office or the patient's house. In the hospital, communication will depend on the place of the meeting, whether a hospitalization ward or a private room. The clinical space also has its own protocols, which contribute to a better communication.

Choosing the place where we will meet with a child or adolescent and their family, preparing for such meeting, and adhering to professional rules are just some important steps that will benefit our patients. Let us not forget that we are not friends with our patients and that we will never take their parents' role, but we may make huge contributions to their well-being and improve their quality of life through empathy, understanding, and dialog. The "humanistic brain", which never stops improving thanks to face-toface meetings with patients and their families, thanks to reading, art, and life, will never be replaced by technology.

\section{Fernando Álvarez \\ Professor of Pediatrics CHU-Sainte Justine University of Montreal, Canada}

http: / / dx.doi.org/10.5546/ aap.2021.eng.220

To cite: Álvarez F. The past, present, and future role of pediatricians. Arch Argent Pediatr 2021;119(4):220-221.

\section{Prohibition of the attachment bond in the Third Reich}

Andrew Ivy, an expert in American medical ethics sent by the American Medical Association to testify in the Nuremberg Doctors' Trial, stated: "Had the profession taken a strong stand against the mass killing of sick Germans before the war, it is conceivable that the entire idea and technique of death factories for genocide would not have materialized. This sentinel genocide was largely conceived, led, and implemented by health professionals and scientists whose core duty should have been to protect the sick and the socially vulnerable". ${ }^{1}$ A paradigm of such sinister account is embodied by Austrian pediatrician and psychiatrist Hans Asperger (1906-1980), ${ }^{2}$ known for identifying Asperger syndrome, who actively assisted the Nazi regime under the child "euthanasia" program; he sent dozens of children to the child psychiatric clinic Am Spiegelgrund in Vienna, where they were killed due to their "uneducable" psychiatric problems. A total of 789 children were killed there between 1940 and 1945. ${ }^{3}$

In May 2017, almost 100 scholars, educators, and medical professionals from around the world met in Western Galilee, Israel, to discuss medicine during and after the Holocaust. The conference resulted in the drafting of what is now known as the Galilee Declaration, ${ }^{4}$ which affirms the Declaration of the Stockholm International Forum on the Holocaust and supplements it for health professions. It calls upon medical schools and other health care institutions to incorporate the 